\title{
Response to Comments on "A Novel Approach to Determine the Astronomical Vessel Position"
}

Jiang-Ren Chang

Associate Professor, Department of Systems Engineering and Naval Architecture, National Taiwan Ocean University, 2, Pei-Ning Road, Keelung, Taiwan 202, R.O.C., cjr@sena.ntou.edu.tw

Chih-Li Chen

Assistant Professor, Department of Merchant Marine, National Taiwan Ocean University, 2, Pei-Ning Road, Keelung, Taiwan 202, R.O.C.

Tien-Pen Hsu

Associate Professor, Institute of Civil Engineering, National Taiwan University, 1, Sec. 4, Roosevelt Road, Taipei, Taiwan 10617, R.O.C.

Follow this and additional works at: https://jmstt.ntou.edu.tw/journal

Part of the Electrical and Computer Engineering Commons

\section{Recommended Citation}

Chang, Jiang-Ren; Chen, Chih-Li; and Hsu, Tien-Pen (2004) "Response to Comments on "A Novel Approach to Determine the Astronomical Vessel Position"," Journal of Marine Science and Technology. Vol. 12: Iss. 2, Article 9. DOI: $10.51400 / 2709-6998.2230$

Available at: https://jmstt.ntou.edu.tw/journal/vol12/iss2/9

This Research Article is brought to you for free and open access by Journal of Marine Science and Technology. It has been accepted for inclusion in Journal of Marine Science and Technology by an authorized editor of Journal of Marine Science and Technology. 


\title{
Communication
}

\section{RESPONSE TO COMMENTS ON "A NOVEL APPROACH TO DETERMINE THE ASTRONOMICAL VESSEL POSITION"}

\author{
Jiang-Ren Chang, ${ }^{*}$ Chih-Li Chen, ${ }^{* *}$ and Tien-Pen Hsu***
}

In response to the comments regarding our recently published paper in the Journal of Marine Science and Technology [3], we would like to begin by expressing our appreciation to Professor Yves Robin-Jouan for his positive remarks. After careful consideration, we offer several points, as follows.

Originally, due to the necessities of teaching, we found that some formulae, which appear in spherical trigonometry, could be reinterpreted using vector algebra, and could further be applied to resolve two classical navigational problems: the great circle sailing (GCS) and the astronomical vessel position (AVP). We made a chance discovery that the geometrical properties of spherical triangles could be formulated in a vector form. Thus, by using the trigonometric equation solving technique to yield these theoretical formulae, they can be applied to construct direct calculation methods, namely the Simultaneously Equal-altitude Equations Method (SEEM) and the Great Circle Equation Method (GCEM), respectively [3, 4]. Aimed at the methodology for solving AVP problems, the available alternatives can generally be categorized into the spherical triangle method and the matrix method. Since the matrix is a vector formulation, the SEEM can be considered a matrix method. In fact, the SEEM is formulated using a fixed coordinate system and relative meridian concept, in conjunction with vector algebra, to deal with the AVP problem. Therefore, simultaneous equal-altitude equations can be constructed as:

Paper Submitted 05/17/04, Accepted 06/07/04. Author for Correspondence: Jiang-Ren Chang. E-mail: cjr@sena.ntou.edu.tw.

*Associate Professor, Department of Systems Engineering and Naval Architecture, National Taiwan Ocean University, 2, Pei-Ning Road, Keelung, Taiwan 202, R.O.C.

**Assistant Professor, Department of Merchant Marine, National Taiwan Ocean University, 2, Pei-Ning Road, Keelung, Taiwan 202, R.O.C.

***Associate Professor, Institute of Civil Engineering, National Taiwan University, 1, Sec. 4, Roosevelt Road, Taipei, Taiwan 10617, R.O.C.

$$
\begin{aligned}
& \cos d_{1} \cdot \cos t_{1} \cdot \cos { }_{1}+\sin d_{1} \cdot \sin L=\sin H_{1}, \\
& \cos d_{2} \cdot \cos t_{2} \cdot \cos { }_{2}+\sin d_{2} \cdot \sin L=\sin H_{2} .
\end{aligned}
$$

Based on the concept that the observer is exactly the zenith and the celestial sphere is a unit sphere, the length of the unit vector is equal to 1 for geometric definition purposes; the observed altitude of the zenith should be 90 degrees for celestial navigation. This means that:

$$
\cos ^{2} L+\sin ^{2} L=1
$$

The above set of three equations is the "prototype" of the matrix method. However, the trigonometric equation solving technique, from which the other alternatives originated from the matrix method do not adopt, is introduced to solve the AVP. More specifically, the equation representing the relationship of the meridian angles of the two celestial bodies can be calculated first, followed by introduction of the products of trigonometric function. The direct computation equation can be derived as follows:

$$
\cos \left(2 t_{2}+\alpha+\beta\right)=\cos (\alpha-\beta)-\frac{2 E^{2}}{R S} .
$$

Thus, the longitude of the AVP can be determined. In addition, by introducing this additional formula into Eqs. (1) and (2), respectively, one can easily obtain the latitude of the AVP. Moreover, the results can be checked with each other for validation. Due to this characteristic, no singularity problems exist in the SEEM. As for the Method of Coplanar Vertices, we are heartened to know that this method is also effective in solving the singularity problem.

The Method of Coplanar Vertices is very simple to use; however, the translation of spherical to Cartesian coordinates at the beginning, and the reverse at the 
end, are still necessary. Such conditions are not encountered when using the SEEM; we used the SEEM to demonstrate the exercises in class, with only a calculator to solve the whole process. Tables 4 and 8 show the solving process for examples 1 and 2, respectively [3].

In the spherical triangle method, the solving procedures of different alternatives have various formulae suggested for the computation steps [5, 7]; thus, users of these methods are often confused, since the criteria are hard to follow. Therefore, based on minimum error propagation and simplicity criteria for the computation, the optimal formulae, such as the side cosine formula and the four-part formula, are suggested as the calculation procedural steps. This procedure also offers only one "way" from one of the two celestial bodies to determine the AVP, and the exact AVP needs an artificial judgment, within a set of possible AVPs for potential users. If another "way" can be implemented, using the same procedure, such as starting from the counterpart of the celestial body, a different set of parallactic (position) angles with respect to this counterpart may be possible, and may result in other possible AVPs for artificial judgment. The true AVP, however, must exist both ways, simultaneously, and the spurious AVPs can then be automatically filtered out, with no artificial judgment. Hence, a checking procedure can be constructed to determine the true AVP [2]. It should be noted that both the side cosine and the four-part formulae are basic formulae, referred to as genetic codes, in

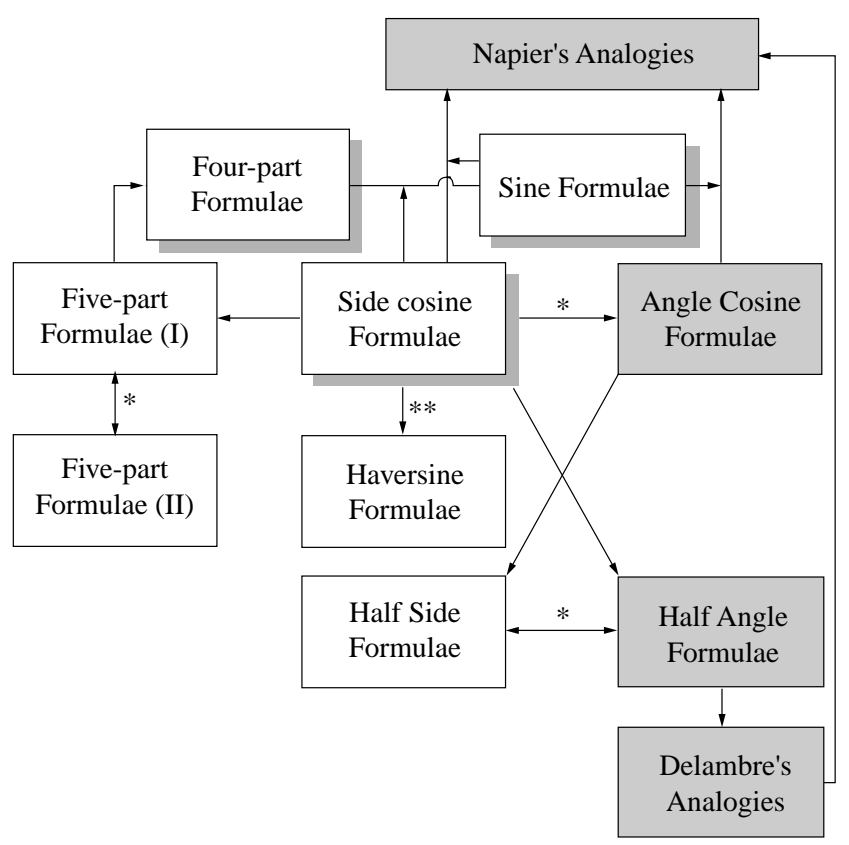

Note: *Polar duality theorem; **Equivalent.

Fig. 1. Relationships of various formulae in spherical trigonometry. spherical trigonometry [6]. The relationships between these various formulae, in spherical trigonometry, are shown in Fig. 1. Incidentally, we actually had not heard of the Paoll method, which uses cosine and cotangent forms of the Gauss formulae; however, if these two formulae are Delmbre's or Napier's analogies, then, because of the perfect alternative symmetry of their formulations, from a mathematical point of view, they sound quite appealing. Nevertheless, in our opinion, they are not as straight-forward, in practical usage, when compared to the genetic code.

Basically the SEEM is only available for the classical problem of celestial navigation in its simplest form, called the condition of the altitude of two celestial bodies, observed at a known GMT, on a known date. For the single celestial body problem, the commonly used intercept method is essentially trial-and-error. To overcome the drawbacks of this method, an improved approach with a new computation procedure has been presented in order to determine the AVP effectively; the results can also be used to successfully demonstrate the SEEM [3]. For the matrix method, used for the problem involving two celestial bodies, a quadratic equation should be encountered when the plane analytic geometry is used [8]; thus, the singularity problem arises. However, the SEEM can avoid this difficulty because this method does not require coordinate transformations. It should be noted that the key point of the SEEM is focused on the solution of the meridian angle. It may appear "tedious" in its forms of expression, but is actually quite straightforward for users familiar with trigonometric equations. We consider questions 4 and 5 to be an "over-determined problem" and have now devoted ourselves to dealing with it.

We wish to extend our sincere thanks for your validation of the SEEM with your Astrolab. It should be noted that when considering the "running fix", the SEEM adopts the geographic position (GP) of the celestial body, but not the EP. In addition, the SEEM provides a transportation facilities function in its solving procedures. Finally, to end our responses, we present the following excerpt from the writings of the great French mathematician, Charles Hermite; after completing a series of research works [2-4], these words [1] popped into our minds.

"I imagine a triangle, although perhaps such a figure does not exist and never has existed anywhere in the world outside my thought. Nevertheless this figure has a certain nature, or form, or determinate essence which is immutable or eternal, which I have not invented and which in no way depends on my mind. This is evident from the fact that I can demonstrate various properties of this triangle, for example that the sum of its three interior angles is equal to two right angles, that 
the greatest angle is opposite the greatest side, and so forth. Whether I desire to or not, I recognize very clearly and convincingly that these properties are in the triangle although I have never thought about them before, and even if this is the first time I have imagined a triangle. Nevertheless no one can say that I have invented or imagined them."

\section{REFERENCES}

1. Bell, E.T., "The Man, Not the Method-Hermite," Men of Mathematics, pp. 448-465, Simon and Schuster, Inc., New York (1937).

2. Chang, J.R., Chen, C.L., and Hsu, T.P., "New Computational Methods for Solving Problems of the Astronomical Vessel Position," Submitted to The Journal of Navigation (2004).

3. Chen, C.L., Hsu, T.P., and Chang, J.R., "A Novel Approach to Determine the Astronomical Vessel
Position," J. Marine Sci. Technol., Vol. 11, No. 4, pp. 221-235 (2003).

4. Chen, C.L., Hsu, T.P., and Chang, J.R., "A Novel Approach to Great Circle Sailings: The Great Circle Equation," J. Navigat., Vol. 57, No. 2, pp. 311-325 (2004).

5. Chiesa, A. and Chiesa, R., "A Mathematical Method of Obtaining an Astronomical Vessel Position," J. Navigat., Vol. 43, pp. 125-129 (1990).

6. Hsiang, W.Y., "Parallelism and Trigonometry," Mathmedia, Vol. 13, No. 2, pp. 14-36 (1989) (in Chinese).

7. Kotlaric, S., "New Short Method Table (K11) for Direct Finding of a Two-Star Fix Without Use of Altitude Difference Method," Navigation, J. Inst. Navigat., Vol. 18, No. 4, pp. 440-449 (1971).

8. Van Allen, J.A., "An Analytical Solution of the Two Star Sight Problem of Celestial Navigation," Navigation, J. Inst. Navigat., Vol. 28, No. 1, pp. 40-43 (1981). 


\section{Communication}

\section{COMMENTS TO ARTICLE IN JMST VOL. 11, NO. 4, PP. 211-235}

From: Yves Robin-Jouan

To: b0037@mail.ntou.edu.tw

Cc: cjr@sena.ntou.edu.tw

Sent: Sunday, April 25, 2004 2:02 AM

Subject: Comments to your article in JMST, Vol. 11, No. 4, pp. 211-235

Hello,

I recently discovered this article, through a selective inquiry concerning "Astrolab" on the web.

This is a very interesting article, indeed.

I have a number of comments about it:

1. My Method of Coplanar Vertices, used in the French "Astrolab" package, has been published in 1995 (thank you for your reference to it). Based on "new" geometric properties, it is more powerful than the classical matrix method, which has been introduced by Dr. Georges Bodenez in 1976 (officially registrated in 1977). Noticeably, Coplanar Vertices solve the well-known singularity problems raised by matrix methods. See my contribution to "Navigation IFN" (Jan 2004, in French...sorry !).

2. The Method of Coplanar Vertices is very simple to use, even by hand : except for the translation of spherical to cartesian coordinates at the beginning and the reverse one at the end, the calculation can be made without a computer. In training sessions with French Paris Observatory, I am used to demonstrate such exercices by hand, for the predictive step of the method. It is much simpler than the tedious trigonometric computations proposed by your SEEM.

3. Concerning direct trigonometric methods, I think that the best one is that of Commander Jean Paoli, published in "bulletin de l'Amicale des Professeurs de l'Enseignement Maritime", N 23, Feb 1995. It uses cosine and cotangent forms of Gauss formulas. And its main advantage is to remove every angle determination problem: better for instance than Charles T. Dozier (before Kotlaric) or Stanley W. Gery alternatives, which are using more classical cosine and sine forms. Do you know Paoli method?

4. What do you propose for more than 2 sights reduction with your SEEM? With Astrolab, I take profit of the computer to maximize the number of observations: 3 is better than $2 ; 4$ than 3 , and so on (like GPS with RAIM approach)...

5. If No. 4 is OK: there are critical cases with any trigonometric approach: e.g. what about the case of 3 COP, including 2 not intersecting, due to inaccuracy of observations?

6. About your example No. 2 : applying Astrolab for a fix (without any EP), I found exactly your AVP. But it is quite different when you consider a "running fix" between your 2 EP. Astrolab integrates transportation facilities, and is consequently able to deliver the resulting "good" position. Do you provide such facilities with SEEM ?

Thank you very much for an expected answer,

Best regards

Yves Robin-Jouan

NAVECOM, Director

IFN \& ION Member 